Abbreviations:

$\mathrm{PL}=$ Plenary $; \quad \mathrm{OP}=$ Oral presentation $; \mathrm{LO}=$ Lightning oral;

$\mathrm{MP}=$ Moderated poster; $\mathrm{P}=$ Poster

*Corresponding authors are underlined.

\section{Plenary Oral Presentations}

\section{PL001}

Derivation of a 2-hour high-sensitivity troponin $T$ algorithm for rapid rule-out of acute myocardial infarction in emergency department chest pain patients

A. McRae, MD, Y. Ji, PhD, H. Yang, MSc, D. Southern, MSc, D. Wang, MSc, I. Seiden-Long, PhD, L. DeKoning, PhD, P. Kavsak, PhD, E. Lang, MD, G. Innes, MD, M. Graham, MD, J. Andruchow, MD, MSc; University of Calgary, Calgary, $\mathrm{AB}$

Introduction: Chest pain and symptoms of acute coronary syndrome are responsible for a large proportion of ED visits and acute hospitalizations. However, only about $15 \%$ of patients presenting to the ED with high-risk symptoms do, in fact, have an acute coronary syndrome. The objective of this study is to derive a 2-hour high-sensitivity Troponin T (hsTnT) testing algorithm with outcome based-cutoffs to rapidly rule out acute myocardial infarction (AMI) in a large proportion of ED chest pain patients. Methods: Patients included consecutive ED patients with a chief complaint of cardiac chest pain who had an hsTnT assay performed at ED arrival and 2 hours after ED arrival. Administrative databases were queried to identify troponin results and major adverse cardiac outcomes (MACE) including death, MI, and revascularization. Test characteristics of iterative combinations of initial troponin level and absolute change in troponin level were quantified in order to identify the testing algorithm that identified the greatest proportion of patients eligible for early discharge while maintaining a target sensitivity of $98.5 \%$ for the primary outcome of 7-day AMI. Results: 755 eligible patients had hsTnT assays performed at ED arrival and at 2 hours. 91 patients (12.1\%) had a 7-day AMI while 108 (14.0\%) had 7-day MACE. An initial hsTnT level of less than $14 \mathrm{ng} / \mathrm{L}$, in combination with a 2-hour absolute change of less than $10 \mathrm{ng} / \mathrm{L}$ had a sensitivity of $98.9 \%(95 \%$ CI $94.0,99.8)$ and an NPV of 99.8\% (95\% CI 98.7, 100.0) for 7-day AMI. This identified 58.5\% of all patients as being suitable for early discharge. Sensitivity and NPV for 7-day MACE were $90.0 \%$ (95\% CI 83.3, 94.2) and 97.3\% (95\% CI $95.3,98.4)$ respectively. Sex-specific differences in test characteristics were not clinically important. Rule-in hsTnT cutoffs were also evaluated, with specificities ranging from $85-95 \%$, although cutoffs with higher specificity had less ability to rapidly rule-in AMI, leaving more patients with indeterminate results after 2 hours. Conclusion: A hsTnT algorithm can safely and accurately rule out AMI in $58.5 \%$ of ED chest pain patients within 2 hours of ED arrival. The lower sensitivity of this algorithm for MACE compared to AMI speaks to the importance of clinical assessment and ECG findings in identifying patients at risk for acute coronary syndromes.

Keywords: troponin, acute coronary syndromes, acute myocardial infarction

\section{PL002}

A randomized controlled trial on oral analgesic utilization for children presenting with a musculoskeletal trauma in the emergency department
S. Le May, RN, PhD, S. Ali, MDCM, A. Plint, MD, B. Mâsse, PhD, G. Neto, MD, M. Auclair, S. Gouin, MDCM; CHU Ste-Justine, Montreal, QC

Introduction: Background: A single-agent approach to children's moderate to severe pain is often inadequate. To date, no studies have evaluated the combined use of oral morphine and ibuprofen for optimal pain management of children presenting to an Emergency Department (ED) for musculoskeletal (MSK) trauma. Objective: To assess the efficacy of a combination of oral morphine and ibuprofen for pain management in children with MSK trauma in the ED. Methods: A double-blind, placebo-controlled, multi-centered, threearm, randomized clinical trial of 500 patients was conducted at three pediatric tertiary care EDs. Patients 6 to 17 years of age, who presented to the ED with a MSK trauma, and a score $>30 \mathrm{~mm}$ on the $100 \mathrm{~mm}$ Visual Analogue Scale were eligible to participate. Patients were randomized (in a 2:1:1 ratio) to receive (orally): (a) morphine $(0.2 \mathrm{mg} / \mathrm{kg})+$ ibuprofen $(10 \mathrm{mg} / \mathrm{kg})$ (Group MOR + IBU) or (b) morphine $(0.2 \mathrm{mg} / \mathrm{kg})$ + placebo (Group MOR) or (c) ibuprofen $(10 \mathrm{mg} / \mathrm{kg})+$ placebo (Group IBU). Primary outcome was pain intensity score under $30 \mathrm{~mm}$ (mild pain) at 60-minutes (T-60) after treatment administration. Results: A total of 456 patients were included in analyses: 177 (MOR + IBU), 188 (MOR), 91 (IBU). Mean age was $11.9+2.7$ years, with a majority of boys $(55.3 \%)$ and soft tissue injuries (62\%). There were no differences in baseline characteristics in the three groups. Baseline mean pain score was $60.9+16.2 \mathrm{~mm}$. Only 30\% (MOR + IBU), 29\% (MOR) and 30\% (IBU) of patients reached a pain score under $30 \mathrm{~mm}$ at T-60 $(\mathrm{p}=0.83)$. Mean pain scores at T-60 were $42.3+23.2 \mathrm{~mm}$ (MOR + IBU), $43.8+23.1 \mathrm{~mm}$ (MOR) and $42.3+23.3 \mathrm{~mm}$ (IBU) $(\mathrm{p}=0.83)$. No severe adverse events were observed in any of the groups, at any of the study measurement points. Conclusion: Combination of morphine with ibuprofen did not provide any additional pain relief for children with MSK injuries, in the ED. None of the study medication provided optimal pain management, as the majority of children did not reach the WHO definition of mild pain. Alternative analgesic combinations should be investigated to optimize pain relief of children who present to the ED with MSK injuries.

Keywords: pain, pediatric, clinical trial

\section{PL003}

Impact of process improvements on measures of emergency department efficiency

A. Leung, MD, M. Duic, MD, D. Gao, MSc, S. Whatley, MD; Southlake Regional Health Centre, Newmarket, ON

Introduction: The objective was to study the operational impact of an intervention comprised of simultaneous process improvements to triage, patient inflow, and physician scheduling patterns on emergency department (ED) patient flow. The intervention did not require any increase in ED resources or expenditures. Methods: A 36-month pre-/post-intervention retrospective chart review at an urban community emergency department from January 2010 to December 2012. The ED process improvements started on June 6, 2011 and involved streamlining triage, parallel processing, flexible nurse-patient ratios, flexible exam spaces, and flexible physician scheduling. The main outcomes were ED length-of-stay (LOS). Secondary outcomes included time to physician-initial-assessment (PIA), left-without-being-seen (LWBS) rates, and left-against-medical-advice (LAMA) rates. Segmented regression of interrupted time series analysis was performed on Canadian Triage and Acuity Scale (CTAS) 2 to 5 patients to quantify 